Journal of

Anesthesia and Surgical Care

\title{
Ultrasound-Guided Evacuation of A Perineural Abscess: Illustrating Anesthesi- ologist's Skills Beyond Anesthesia Practice
}

\author{
Deborah Lambert $\mathrm{MD}^{1}$, Nassim Touil $\mathrm{MD}^{1}$, Arnaud Potié $\mathrm{MD}^{2}$, Bernard le Polain de Waroux $\mathrm{MD}^{1}$ Mona \\ Momeni, $\mathrm{MD}, \mathrm{PhD}^{1}$, Veevaete Laurent $\mathrm{MD}^{1,3 *}$
}

${ }^{1}$ Department of Anesthesiology, Cliniques Universitaires Saint-Luc, Université catholique de Louvain, Brussels, Belgium

${ }^{2}$ Department of Anesthesiology, Centre Hospitalier Universitaire Vaudois, Lausanne, Switzerland

${ }^{3}$ Department of Anesthesiology, Clinique Saint-Pierre, Ottignies, Belgium

${ }^{\star}$ Corresponding author: Laurent Veevaete, MD, Department of Anesthesiology, Clinique Saint-Pierre, Avenue Reine Fabiola 9, 1340 Ottignies, Belgium; e-mail: veevaetelaurent@gmail.com

Received Date: June 13, 2019 Accepted Date: September 02, 2019 Published Date: September 04, 2019

Citation: Deborah Lambert (2019) Ultrasound-Guided Evacuation of A Perineural Abscess: Illustrating Anesthesiologist's Skills Beyond Anesthesia Practice. J Anesth Surg Care 1: 1-4.

\begin{abstract}
Ultrasonography (US) is nowadays widely used by anesthesiologists while performing locoregional anesthesia. The use of US beyond loco-regional anesthesia is becoming frequent $[1,2,3,4]$. We describe a case of US guided perineural abscess evacuation performed by an anesthesiologist familiar with loco-regional anesthesia.
\end{abstract}

Keywords: Catheter; Continuous Peripheral Techniques; Diagnostic Imaging; Perineural Abcess.

\section{Introduction}

Nowadays, loco-regional anesthesia guided by ultrasound is a prevalent practice, which is especially used for the placement of perineural catheters with the aim to provide analgesia. Its use beyond perineural catheter placement is not frequently described.

We report a case of US guided perineural abscess evacuation performed by an anesthesiologist, familiar with loco-regional anesthesia. For publication of this report, the patient's written consent was obtained.

\section{Description}

A popliteal sciatic perineural catheter had been inserted by the Pain Service under ultrasound guidance in a 54-year-old man (ASA III) suffering from severe foot pain.

C2019 The Authors. Published by the JScholar under the terms of the Creative Commons Attribution License http://creativecommons.org/licenses/ by $/ 3.0 /$, which permits unrestricted use, provided the original author and source are credited.
The patient's history included a medical history of diabetes mellitus type 2 without insulin, an atrial fibrillation, a pacemaker and an obstructive sleep apnea.

The patient's weight was 66kg and he had a Body Mass Index of 22

The popliteal catheter was inserted in aseptic conditions. The operator was wearing a hat, mask, sterile gown and sterile gloves. The affected area had been disinfected with chlorhexidine. Sterile fields were set up by the anesthesiologist and ultrasound probe protection was used.

A first bolus of ropivacaine $0.2 \%$ was injected and the catheter was connected to a pump with the following program setting: continuous infusion of $5 \mathrm{~mL} / \mathrm{h}$, a bolus of $5 \mathrm{~mL}$ with a refractory period of 50 minutes.

A daily evaluation of the pain relief due was performed by a team specialized in pain management. The catheter was kept in place during 3 weeks to relieve patient's foot pain. It was then removed due to leakage and local site infection. 
The patient was scheduled for foot surgery the day after the catheter removal. While performing US in view of performing a popliteal sciatic nerve block, a hypo-echogenic collection was visualized adjacent to the sciatic nerve (figure 1). This collection was not present while performing the first US 3 weeks earlier.
The decision was made to evacuate the collection with a 18-gauge Tuohy needle (B. Braun, Germany) under high-frequency (6$13 \mathrm{MHz}$ ) linear array ultrasound transducer (SonoSite S-Nerve, Bothell, WA, USA) (figure 2, video). The evacuation was followed by washing out the collection with $10 \mathrm{~mL}$ sterile saline solution repeated four times (figure 3).

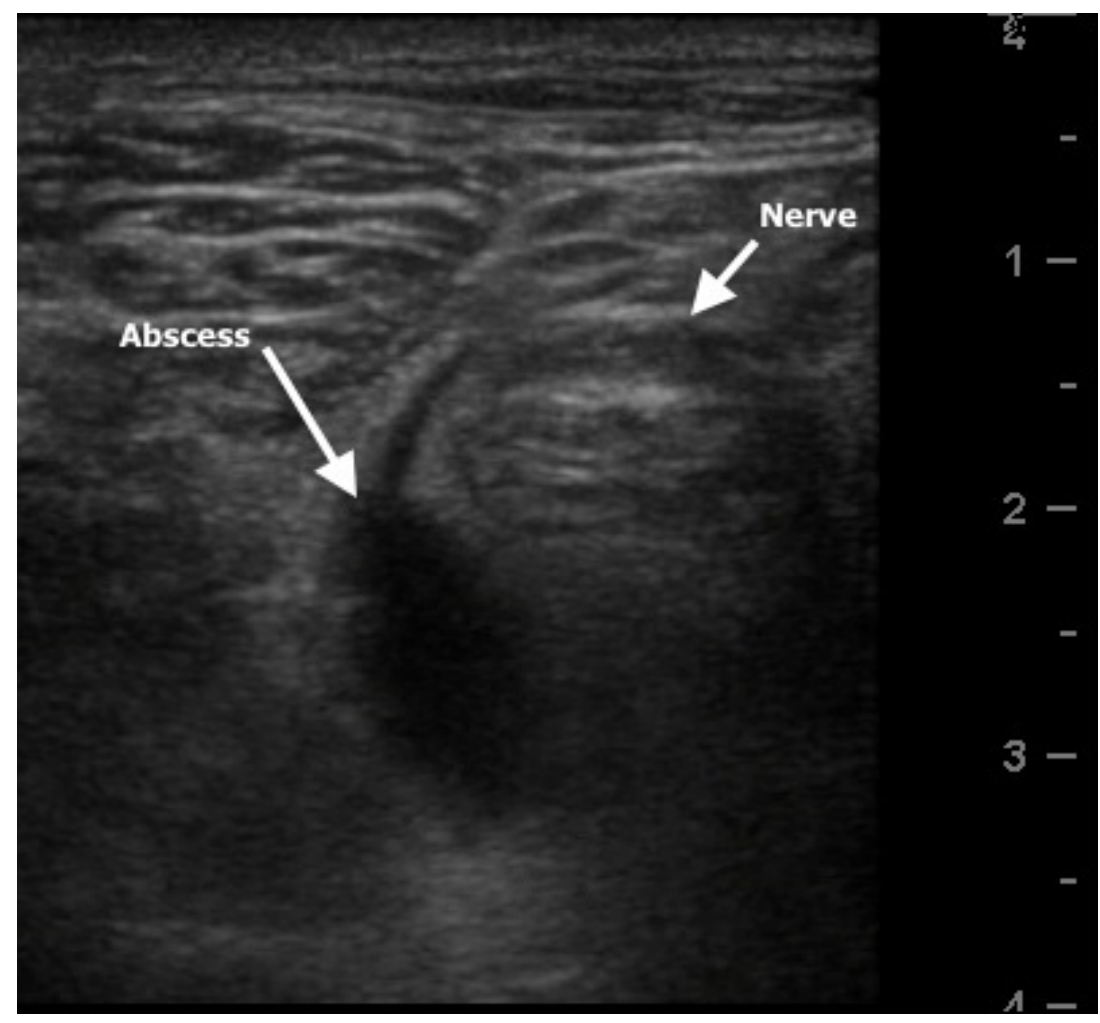

Figure 1: Hypo-echogenic collection was visualized adjacent to the sciatic nerve.

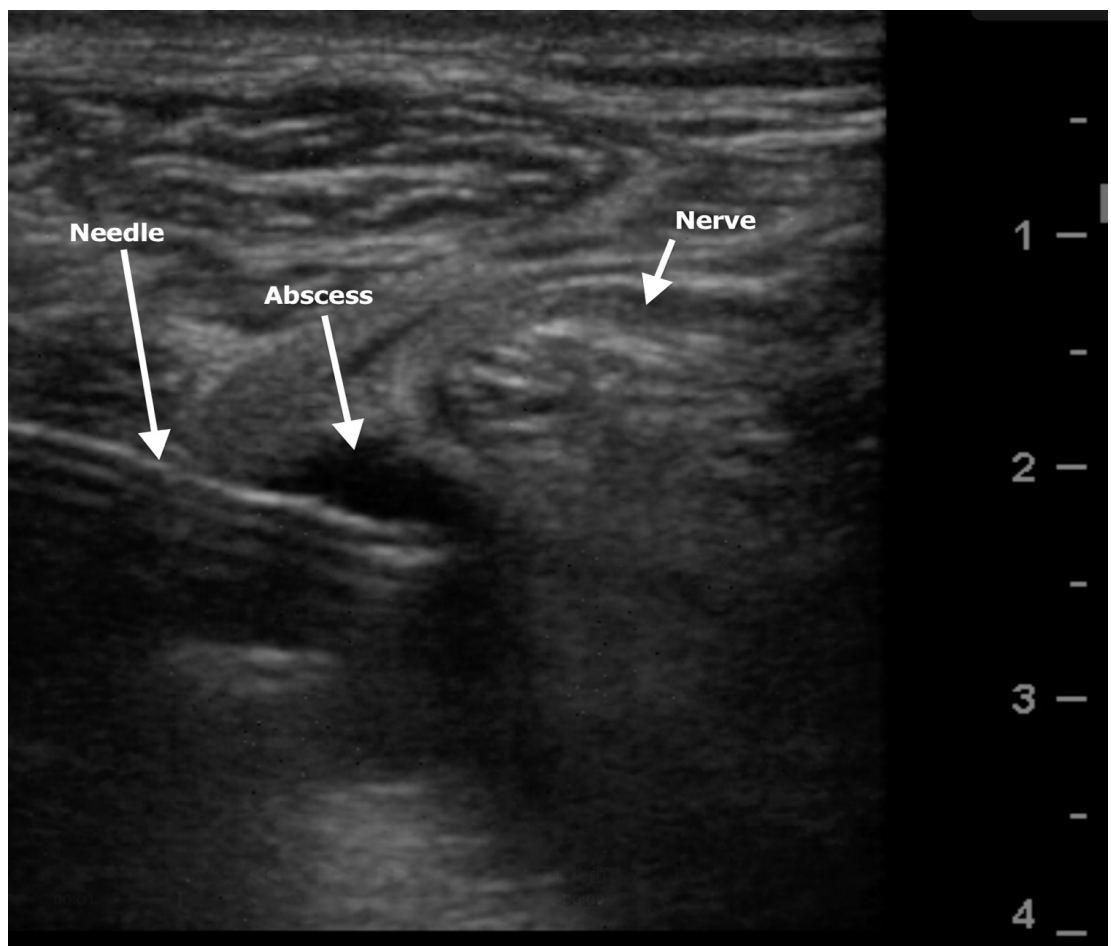

Figure 2: Evacuation of the collection with a 18-gauge Tuohy needle (B. Braun, Germany) under high-frequency (6-13MHz) linear array ultrasound transducer (SonoSite S-Nerve, Bothell, WA, USA). 


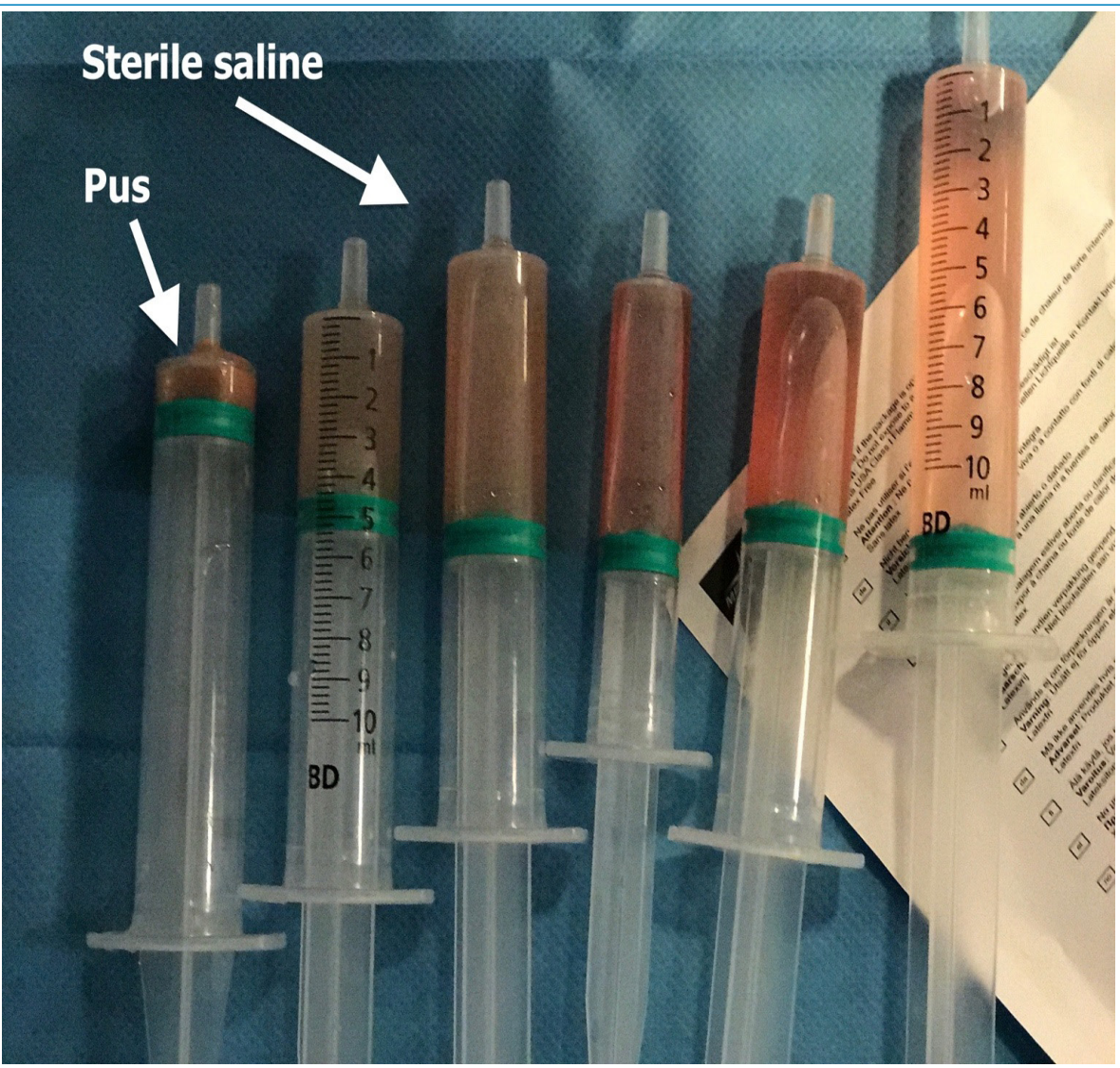

Figure 3: The syringes used to wash the collection. The collected pus is in the left one and the last rinse is in the right one.

Considering the risk-benefit ratio for the patient, the surgery was performed under subgluteal sciatic nerve block without catheter.

Bacteriological examination of the evacuated collection revealed Pseudomonas Aeruginosa and Corynebacterium Striatum. Antibiotic treatment was started with intravenous Piperacilline-Tazobactam four times a day during the following 10 days.

The post-operative follow-up of the patient was from a surgical point of view uneventful and the bacteriological evolution was positive and allowed the patient to leave the hospital two days after his surgery. No signs of local or general secondary infection were visible after this episode.

\section{Discussion}

To our knowledge this is the first time a popliteal collection is drained under echographic guidance by an anesthesiologist. In the literature, only few cases of peri nervous abscesses occurring with catheter placement are described, and every each of them were surgically drained [5]. This case illustrates that echography performed by anesthesiologists can be used as a way to detect complications linked to catheter insertion as well as the treatment of these complications.

There is a difference between catheter colonization, inflammation and infection. Bacterial colonization is microbial growth on catheter tip culture. The signs of inflammation at the catheter entry site are erythema, itchiness or warmness. Catheter infection is defined as signs of systemic infection such as fever, leucocytosis, elevated infection-inflammation markers and positive blood culture, in a patient with signs of catheter entry site inflammation or evidence of abcess [6]. A meta-analysis [7] of randomized controlled trials showed that catheter colonization occurred in 6 to $69 \%$ of cases with some signs of inflammation in 3 to $9.6 \%$ and with signs of infection in 0 to $3 \%$. Neuburger et al. [6] proposed that inflammation could be related to tissue trauma due catheter movement. Three possible explanations are proposed for peripheral nerve block infection : bloodstream diffusion, contamination of infused drugs and pathogen penetration through catheter entry [6]. It seems that the formation of a hematoma close to the insertion site of the catheter is a possible risk factor for the development of an infection. Therefore, some experts recommend to remove the catheter in the case of hematoma adjunctant to the puncture site [6]. In our patient, the 
inflammation markers were high. However, this can be due to catheter infection or be a consequence of his diabetic foot.

Infectious complications of perineural catheters are rarely reported in the literature, estimated at $2.4 \%$, and only few cases report sepsis $[5,6,7,8]$. Lethal infection was described in one case [6].

Risk factors for bacterial colonization of perineural catheters are the following: $[5,6,7,8]$

- patient's comorbidities (diabetes, corticotherapy, febrile seizure, conditions of mild to overt immunosuppression)

- site of catheter insertion (femoral, axillary, interscalene block)

- catheters in place for more than $48 \mathrm{~h}$

- the use of non-alcoholic antiseptic solution

- a local anesthetic extemporaneously reconstituted

- the absence of prophylactic antibiotic treatment

- antibiotic administration during the month prior to surgery

- trauma victims

- sepsis

- hospitalization in an intensive care unit

- frequent changing of dressing

- postoperative hyperglycemia.

Our patient presented two of these risk factors which are diabetes and the catheter which had been left in place for more than $72 \mathrm{~h}$.

In the study conducted by Cuvillon 14 focusing on femoral catheters, bacterial colonization occurred in $57 \%$ of the cases and an incidence of bacteremia of $1.5 \%$ was present after $48 \mathrm{~h}$ of continuous infusion. The most commonly Pathogens involved are the Staphylococcus aureus and the Staphylococcus epidermidis. Escherichia Coli and Enterococcus are reported as well. This reveals a high colonization rate but infection remains rare. Infection occurs by endoluminal contamination of a soiled substance or by cutaneous migration of skin saprophytic bacteria $[5,6,7,10]$.

A retrospective study [11] dealt with the use of perineural catheters on patients with arthrite. An infection had been observed in $9.7 \%$ cases and the infection of the popliteous site occured more often during the postoperative analgesia. The pathogens found in $71 \%$ cases were Staphylococcus aureus followed by gram-negative bacilli including two Pseudomonas Aeruginosa, one Serratia and one Escherichia Coli. Two types of patients were distinguished: infection after a short period of infusion and infection after a long period of infusion (55 days on average). In this study, the average duration of catheter use was 35 days. However, the authors suggest a maximum of three days of continuous infusion with the same local anaesthetic container.

Another important point is the preventive measures required to decrease infections. The insertion of the catheter under surgical aseptic techniques is very important. The guidelines recommend to isolate the target site with sterile drapes and to wear sterile gloves, sterile gown, hat and mask and to use antiseptic dressing, antiseptic solution for the disinfection and antimicrobial filters. The first choice of the skin disinfectant between iodine and chlorhexidine is on chlorhexidine gluconate that penetrates through the lipid layer in the skin and which is more effective and rapid in eliminating skin flora than povidone iodine [6]. It has been shown that the use of tunneled catheters would reduce infection emergence $[6,8,12]$. To reduce the risk of infection, avoiding hyperglycemia in the postoperative period is another important point although no consensus exists on glycemia targets [6]. In our patient, the aseptic guidelines for the placement of the catheter were respected but the catheter was not tunneled.

\section{Conclusions}

To our knowledge, this is the first case of localized treatment of an abscess performed by an anesthesiologist expert in ultrasound-guided locoregional anesthesia. This case also illustrates the importance of performing ultrasound in daily anesthesia practice.

To conclude, it seems important to suggest some recommendations of good practice to insert peri-nervous catheter and to take care of potential complications.

It is recommended to apply the basic precautions like wearing a mask, a hat, sterile gloves, a sterile gown but also to disinfect the area with chlorhexidine and to use an ultrasound probe protection.

It is suggested to remove the catheter as soon as possible (ideally maximum 72 hours after insertion). The patient must be visited everyday during his hospitalization in order to check his 
parameters and to look for any signs of local infection. In case of the detection of infection the catheter must be removed.

An echography control could be made to detect a prospective abscess in the area of puncture point. It would also be interesting to consult an infectious disease doctor to get his advice regarding antibiotherapy.

If an abscess is discovered, practicing this less invasive technique could give more advantages than surgical act: it requires no incision and thus no problem of healing, and provides a lower level of fear/stress for the patient. More investigations are required before recommending this practice.

\section{Contributorship}

Conception or design of the work: all the authors

Data collection: Dr Lambert, Dr Touil, Dr Potié and Dr Veevaete

Drafting the article: Dr Lambert and Dr Touil

Critical revision of the article: all the authors

Final approval of the version to be published: all the authors

\section{References}

1. Pirotte T, Brui B (2006) Ultrasound-guided punctures in anesthesia. Acta Anaesthesiol Belg 57: 401-407.

2. Van de Putte P, Perlas A (2014) Ultrasound assessment of gastric content and volume. Br J Anaesth. 113:12-22.

3. Haskins S, Tanaka C, Boublik J, Wu C, Sloth E (2017) Focused Cardiac Ultrasound for the Regional Anesthesiologist and Pain Specialist, Reg Anesth Pain Med 42: 632-644.

4. Kruisselbrink R, Chan V, Cibinel GA, Abrahamson S, Goffi A (2017) I-AIM (Indication, Acquisition, Interpretation, Medical Decision-making) Framework for Point of Care Lung Ultrasound. Anesthesiology 127: 568-582.

5. Ceron P, Iselin I, Hoffmeyer P, Fournier R (2014) Cervical abcess complicating an ultrasound-guided interscalene catheter. A A Case Rep 3: 53-55.

6. Nicolotti D, Iotti E, Compagnone C (2016) Perineural catheter infection : a systematic review of the literature. J Clin Anesth 35:123-128.
7. Joshi G, Gandhi K, Shah N, Gadsden J, Corman S (2016) Peripheral nerve blocks in the management of postoperative pain : challenges and opportunities. J Clin Anesth 35: 524529.

8. Delfosse F, Pronnier P, Levent T, Coget ML, Blanckaert K (2011) Infectious complicating femoral nerve catheter for postoperative analgesia: about two cases. Ann Fr Anesth Reanim 30: 516-520.

9. Cuvillon P, Ripart J, Lalourcey L, et al. (2001) The continuous femoral nerve block catheter for postoperative analgesia: bacterial colonization, infectious rate and adverse effects. Anesth Analg. 93:1045-1049.

10. Borgeat A, Blumenthal S, Lambert M, Theodoru P, Vienne P (2006) The feasability and complications of popliteal nerve block: a 1001-case survey. Anesth Analg 103: 229-233.

11. Bures E, Rivet P, Steiner T, et al. (2011) From pain to cicatrisation: Perinervous catheter as a treatment for arteritic pain at home for long duration. Douleurs 12: 181-188.

12. Landy C, Polycarpe A, Boulland P, Favier JC, Plancade D (2012) Complications of a continuous popliteal sciatic nerve block. Ann Fr Anesth Reanim 31: 564-573.

Submit your manuscript to a JScholar journal and benefit from:

ฯ Convenient online submission

ฯ Rigorous peer review

ๆ Immediate publication on acceptance

I Open access: articles freely available online

9 High visibility within the field

ฯ Better discount for your subsequent articles

Submit your manuscript at http://www.jscholaronline.org/submit-manuscript.php 